\title{
An Inverse Force Measurement Method to \\ Determine the Injected Structure-Borne Sound \\ Power from an Installation into a Building Element
}

by

Pieter Schevenels, Peter J. G. van der Linden, Gerrit Vermeir

Reprinted from

BUILDING ACOUNALIICS

Volume $17 \cdot$ Number $3 \cdot 2010$ 


\title{
An Inverse Force Measurement Method to Determine the Injected Structure-Borne Sound Power from an Installation into a Building Element

\author{
Pieter Schevenels ${ }^{1,3}$, Peter J. G. van der Linden ${ }^{2}$, Gerrit Vermeir ${ }^{1}$ \\ ${ }^{1}$ K.U.Leuven, Laboratory of Acoustics and Laboratory of Building Physics, \\ Celestijnenlaan 200D, bus 02416, B-3001 Heverlee, Belgium \\ email:pieter.schevenels@bwk.kuleuven.be \\ ${ }^{2}$ Qsources BVBA, Ketelwinning 38, 3293 Diest, Belgium \\ ${ }^{3}$ Belgian Building Research Institute, \\ Avenue Pierre Holoffe 21, 1342 Limelette, Belgium
}

(Received 11 August 2010 and accepted 10 November 2010)

\begin{abstract}
In structure-borne sound problems in buildings, knowledge of the contact forces between vibrating equipment and a receiving element is often required. When multiplying these forces with the simultaneously measured velocities at the contact points of the equipment, the structure-borne sound power can be determined, which is the quantity governing the sound energy transfer from a source space to a receiving space. Measuring contact forces directly is however not straightforward. Therefore, a structural-acoustical method is used to determine the contact forces inversely. This technique is already used in the automotive industry, but applications in buildings are rather rare. The installation used is a vibrating platform, which induces tonal, low-frequency vibrations into the building. Therefore, the used sound source has to perform well for low frequencies. The resulting forces are used for structure-borne sound power calculation and verified by comparing the sound pressure, caused by this injected power, with measured sound pressure.
\end{abstract}

\section{INTRODUCTION}

Structure-borne sound excitation by vibrating equipment in buildings can be an important noise contributor. The sound transmission is quantified on a power balance basis, as depicted in the European Standard EN 12354-5 [1]. The obvious way to determine the injected power of an installation into a building element is through multiplication of velocity and force at each contact point of the installation. The velocity can easily be measured approximately by attaching an accelerometer on the building element close to the contact point. To measure the force however, one would have to insert a force sensor into the path between the installation and the building element, and this is a rather cumbersome task. Also, since the transfer path is physically altered, this might change the power transfer through the path. 
A more convenient way of determining the forces between an installation and a building element is by inverse methods that do not involve changing the transfer path. There exist purely structural methods to do so and these are widely applied. Investigations using structural reciprocity in building related structure-borne sound problems can be found in [2] and [3]. Structural-acoustical method also can be used for this purpose. In this case, first the pressure is measured in a receiving space in an arbitrary point while the installation operates, afterwards the structural-acoustical frequency response function (FRF) of volume velocity and velocity is measured between the arbitrary point and the considered contact point.

This can be done in a direct way by exerting a force in the contact point and by measuring the velocity in the arbitrary point for the structural method or by measuring the pressure in the arbitrary point for the structural-acoustical method. However, it is often easier to determine these FRFs reciprocally, because of lack of place to exert a force correctly and because different FRFs can be measured simultaneously. A system is reciprocal when a FRF from position 1 to position 2 equals the corresponding FRF from position 2 to position 1 . The principle of reciprocity was given in 1860 by Helmholtz for acoustical systems [4]. In 1873, Lord Rayleigh applied the reciprocity concept on various other fields and stated reciprocity is valid for all stable, lumped, linear, passive, dynamic systems which only contain bilateral elements (like masses, stiffnesses, capacitors and resistors) [5]. However, the validity of reciprocity for mechanical and structural-acoustical systems remained uncertain for a long time [6]. The most important application was the reciprocal calibration of microphones and hydrophones, as proposed by Ballantine in 1929 [7]. Lyamshev [8] and Chertock [9] give formal proofs of the validity of reciprocity and ten Wolde published on the validity of reciprocity [10]. This set the door wide open for a lot of applications from 1970 onwards. Recurring names in conference proceedings and articles since then are ten Wolde, Verheij, Fahy and Vér.

In a structural-acoustical system with only 1 contact point $C$ and only a transversal force, reciprocity can be written as follows [11]:

$$
\left.\frac{p_{X}^{\odot}}{F_{C}^{\odot}}\right|_{Q_{X}^{\odot}=0}=-\left.\frac{a_{C}^{\otimes}}{Q_{X}^{\otimes}}\right|_{F_{C}^{\otimes}=0}
$$

where $X$ is an arbitrary point in a space of the building and the symbols $\odot$ and $\otimes$ indicate direct excitation and reciprocal excitation respectively. To determine the unknown force $F_{C}$, a known volume acceleration is generated in point $X$ by a volume acceleration sound source $\left(Q_{X}\right.$ in $\left.\mathrm{m}^{3} / \mathrm{s}^{2}\right)$, while the acceleration $a_{C}$ is measured in $C$ in absence of the installation. Next, the sound pressure is measured in $X$ while the installation is operating $\left(p_{X}\right)$. The force can be deduced from eqn (1) as follows:

$$
F_{C}=-\left(\frac{a_{C}}{Q_{X}}\right)^{-1} p_{X}=-H^{-1} p_{X}
$$

where $H$ is the FRF. 
At an installation with different contact points, a force is present at each contact point. By using a matrix formulation of eqn (2), multiple forces can be calculated as:

$$
\mathbf{F}_{C}=-\mathbf{H}^{-1} \mathbf{p}_{X}
$$

For an installation with 3 contact points $(a, b$ and $c$ ) and when only transversal force components have to be considered, eqn (3) can be written out as:

$$
\left[\begin{array}{c}
F_{C, a} \\
F_{C, b} \\
F_{C, c}
\end{array}\right]=-\left[\begin{array}{lll}
\frac{a_{C, a}}{Q_{X, 1}} & \frac{a_{C, b}}{Q_{X, 1}} & \frac{a_{C, c}}{Q_{X, 1}} \\
\frac{a_{C, a}}{Q_{X, 2}} & \frac{a_{C, b}}{Q_{X, 2}} & \frac{a_{C, c}}{Q_{X, 2}} \\
\frac{a_{C, a}}{Q_{X, 3}} & \frac{a_{C, b}}{Q_{X, 3}} & \frac{a_{C, c}}{Q_{X, 3}}
\end{array}\right]^{-1}\left[\begin{array}{c}
p_{X, 1} \\
p_{X, 2} \\
p_{X, 3}
\end{array}\right]
$$

This means that in the receiving space, the number of points $X$ equals the number of contact points $C$ (see Figure 1). The number of FRFs is the square of this number. For installations with a lot of contact points, this means a lot of work. When moreover other force components like moments become important, the number of FRFs consequently increases significantly.
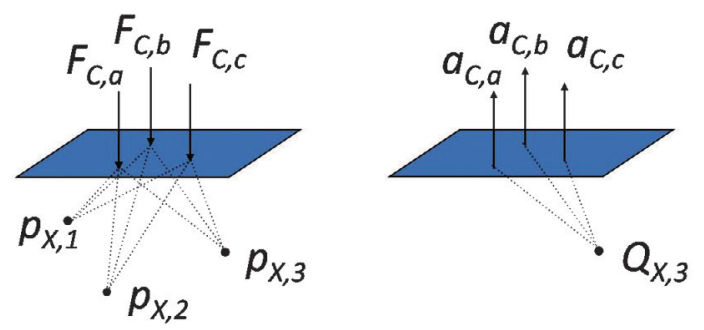

Figure 1. For an installation with transversal forces through 3 contact points, 3 pressures have to be measured in the receiving space (left). The 3 FRFs $a_{C, i} / Q_{X, 3}$ can be determined in 1 measurement (right).

The structural-acoustical reciprocity technique to determine an unknown force is used in noise and vibration research and development in the automobile industry, aviation industry and in naval shipbuilding, but apart from work by Geebelen [12] is rarely used in buildings. The reason is threefold. First, the objects that need to be put into motion are much heavier in buildings than in automobile and aviation industry, setting requirements to the power of the volume acceleration sound source. Secondly, the frequency range of interest in buildings is generally low, due to the nature of building ser- 
vice equipment, and low-frequency volume acceleration sound sources are required. Furthermore, also the positioning of the sound source becomes important when long wavelengths are involved. Thirdly, the recent developments in normalization in the standard EN 12354-5 and the corresponding research efforts play a role; it is only in the last decade that injected structure-borne sound powers and corresponding forces in building structures have become a point of interest.

When the inversely measured forces are multiplied by the velocities in each contact point, the complex structure-borne sound power is obtained. These velocities are measured simultaneously as the pressures used to inversely measure the force. The real part of this power, the injected structure-borne sound power, can been determined as:

$$
W_{i n j}=\frac{1}{2} \operatorname{Re}\left\{\mathbf{F}_{C}^{H} \mathbf{v}\right\}
$$

where the superscript ${ }^{\mathrm{H}}$ denotes the Hermitian transpose, which is the conjugate transposed.

The injected structure-borne sound power is the input parameter for the calculation of the sound pressure in any room of the building, according to EN 12354-5 [1]. This standard states the acoustic behaviour of a building is determined by the properties of its elements, but remains unclear on how to determine the input parameter of the injected structure-borne sound power. A method is however suggested for light-weight sources on heavy-weight building elements and refers to a reception plate method for this special case, outlined in EN 15657-1 [13].

\section{METHODS TO CALCULATE THE FORCE}

Since a matrix inversion is involved in the process, ill-conditioned matrices can cause problems. Errors, that are always present in the measurement of the pressure, will be amplified if the condition number of the FRF matrix is high. Measurement errors in the measurement of the FRFs themselves will of course also yield errors. This is especially a problem for frequency ranges with a small number of eigenmodes, or, equivalently, for installations with the contact points close to each other. The smaller the number of eigenmodes is, the more dependent the FRFs at the different measurement points of the building element will become to each other and the higher is the resulting condition number.

\subsection{Total force}

A way to circumvent problems with matrix calculations is to represent multiple contact points as one contact point of which the total force has to be determined. This results in a scalar problem without the need to invert a matrix. The number of FRFs that has to be measured in this case, equals the number of contact points. These FRFs are averaged and eqn (2) is used with the average of the FRFs. For 3 contact points $a, b$ and $c$, this becomes:

$$
F_{C}=-<\left(\frac{a_{C}}{Q_{X}}\right)^{-1}>_{(a, b, c)} p_{X}=-<H^{-1}>_{(a, b, c)} p_{X}
$$


The disadvantage of this method is of course that no information is available about the forces at each contact point. However, in some cases, knowledge of the exact force distribution is not required. This can happen when the contact points are close to each other. Also, when there are line contacts or surface contacts, determining a total force over that contact is much more sensible than dividing the contact area into arrays of point contacts. In this case, eqn (5) to determine the sound power becomes scalar and the averaged velocity over the contact points has to be determined. This also implies that the phase of the resulting averaged velocity is an average.

\subsection{Separate forces}

When information of separate forces is required however, there are several approaches to the problem of ill conditioned matrices. Most of them are purely mathematical and based on matrix manipulation. Blau looked at the influence of errors in the measurements of the FRF matrix and of errors in the measurements of the response values. He considered arbitrary errors, systematic errors as well as leakage errors [14].

\subsubsection{Over-determination}

A first physical approach is to over-determine the matrix. This means that more FRFs are measured than technically necessary. The resulting matrix $\mathbf{H}$ is $(m, n)$-sized, with $n$ the number of forces and $m>n$ the number of measured FRF functions per force. In the example of structural-acoustical reciprocity this means that $m$ positions are needed where the volume acceleration sound source is placed to determine the FRF $H$. The pressure $p$ is measured at the same $m$ positions when the structure-borne sound source is operating. The degree of over-determination $m-n$ is therefore limited in practice by the available number of microphones and the number of channels on the data acquisition equipment. Since this matrix cannot be inverted in the classical way, a pseudoinversion needs to be applied to find the $(n, m)$-sized pseudo-inverted matrix $\mathbf{H}^{+}=$ $\left(\mathbf{H}^{H} \mathbf{H}\right)^{-1} \mathbf{H}^{H}$.

Researchers have considered the positions most suitable for measurement of the pressures $p$. Provided that this is a good choice, no mathematical techniques need to be applied to improve the condition number because it is already at its smallest. Nelson and Yoon concluded that the sensors (microphones in this case) should be placed close to the (structure-borne sound) source, that the distance between the sensors should be approximately equal to the distance between the forces in the contact points and that the geometry of the sensor locations is similar to and symmetrical in respect to the location of the forces [15]. Thite and Thompson considered a technique based on minimizing the averaged condition number over the frequency range of interest and used the socalled "composite condition number" for this purpose $[16,17]$. They give an overview of several FEM-based techniques, but also describe the purely experimental "reference identification procedure". They conclude that over-determining at low frequencies with a limited number of eigenmodes - does not improve the inversion. Blau also suggested a frequency dependent criterion for location of the sensors [18], but Thite and Thompson argue that this technique is difficult to apply to the whole frequency range of interest [17]. 


\subsubsection{Singular value elimination}

A popular mathematical method to decrease the condition number of a matrix consists of eliminating singular values. Every $(m, n)$-matrix $\mathbf{H}$ can be written as follows [19]:

$$
\mathbf{H}=\mathbf{U} \sum \mathbf{V}^{H}
$$

where $\mathbf{U}$ and $\mathbf{V}$ are squared, orthogonal matrices sized $(m, m)$ and $(n, n)$ respectively and $\sum$ is a $(m, n)$-matrix consisting of a diagonal matrix in the $n$ upper rows and $m-n$ zero rows below if $m>n$. $\mathbf{U}$ and $\mathbf{V}$ consist of the eigen vectors of the operators $\mathbf{H}^{H} \mathbf{H}$ and $\mathbf{H H}^{H}$, also denoted left and right singular vectors, respectively. The diagonal matrix contains the singular values $\sigma_{i}$ on the diagonal, arranged from large to small and all real and larger than or equal to zero.

If the singular value decomposition is applied on the inverse of the FRF matrix $\mathbf{H}^{\mathbf{1}}$ in eqn (3), the matrix approximating the unknown forces $\mathbf{F}_{0}$ can be written as a function of the measured pressures $\hat{\mathbf{p}}$ as follows:

$$
\mathbf{F}_{0}=-\mathbf{V} \Sigma^{+} \mathbf{U}^{H} \hat{\mathbf{p}}
$$

where $\Sigma^{+}$is the pseudo-inverse of $\sum$, sized $(n, m)$ :

$$
\Sigma^{+}=\left[\begin{array}{cccccc}
1 / \sigma_{1} & \cdots & 0 & 0 & \cdots & 0 \\
\vdots & \ddots & \vdots & \vdots & \ddots & \vdots \\
0 & \cdots & 1 / \sigma_{n} & 0 & \cdots & 0
\end{array}\right]
$$

Hence, the smallest singular values (starting from $\sigma_{n}$ ) are responsible for the largest contribution in the matrix with the contact forces $\mathbf{F}_{\mathbf{C}}$. If the condition number - the ratio of the largest singular value to the smallest singular value [20] - is large, inevitable measurement errors in the FRF matrix will result in largely amplified errors in the forces as the absolute error on the smallest singular value gets important. When the smallest singular value is eliminated from the calculation, setting the corresponding values $1 / \sigma_{i}$ in $\Sigma^{-1}$ equal to 0 , the condition number becomes smaller. Measurement errors will thus have less serious consequences. Of course, this has to be organized carefully, because eliminating singular values means eliminating information from the system. Over-elimination gives a matrix, which is not properly representative of the physical system. The result is an underestimation of the unknown contact forces. Moorhouse proposes a compensation method when the measurement errors in the pressures are purely arbitrary and not caused by noise [21].

There are several approaches to eliminate singular values. The simplest is to eliminate the $N$ smallest singular values and to set their inverse equal to 0 . Because the smallest singular value can still be high and not corrupted by noise, this technique is quite rudimentary and the results can be disappointing. A better approach is to set an absolute limit, below which singular values are eliminated. This limit requires analysis in order not to eliminate too many values. It is possible that with all singular values eliminated, 
the force(s) cannot be calculated. It is better to take a relative limit as a ratio in respect to the highest singular value, although again it might be a problem to determine this ratio. An advantage is that at least one singular value is maintained and a force can always be determined. Romano and López propose to eliminate singular values smaller than $10 \%$ of the highest singular value [22].

Another approach is to take the limit relative to the (expected) accuracy of the measurement. Powell suggests the use of the coherence function $\gamma_{i j}^{2}$ as a measure of the expected error in the FRF matrix $\mathbf{H}$ [23]. The error matrix $\mathbf{E}$ has elements $E_{i j}$ [24], where:

$$
E_{i j}=\alpha \sqrt{\frac{1-\gamma_{i j}^{2}}{2 n_{a v} \gamma_{i j}^{2}}}\left|H_{i j}\right|
$$

where $n_{a v}$ is the number of averages used to determine the elements in the FRF matrix H. Hence, $E_{i j}$ equals $\alpha$ times the standard deviation in $H_{i j}$. For $\alpha=3$ and assuming a normal distribution, there is a $99,7 \%$ probability that $H_{i j}$ deviates maximally a value of $E_{i j}$ from the actual value. For $\alpha=1$ [16], some cases yield better results. The global estimate of the error is equal to the norm of $\mathbf{E}$ :

$$
\varepsilon=\|\mathbf{E}\|_{e}
$$

The subscript e denotes the Euclidian norm, which is equal to the 2-norm and is denoted with a subscript 2 . When a singular value $\sigma_{i}<\varepsilon$, the inverse $1 / \sigma_{i}$ is set to 0 . A higher $\alpha$ will therefore result in more singular values that are eliminated from the calculation. Another possible criterion is to eliminate a singular value $\sigma_{i}$ when [25]:

$$
\left(\sigma_{i}+\ldots+\sigma_{n}\right) \leq\|\mathbf{E}\|_{e}
$$

where $n$ is the number of singular values.

Janssens et al. propose a criterion which depends on errors in the response [26]. A singular value $\sigma_{i}$ is eliminated when [16]:

$$
\sigma_{i} \leq \frac{\left\|\sigma_{p}\right\|_{e}}{\|\hat{\mathbf{p}}\|_{e}} \sigma_{1}
$$

where $\sigma_{p}$ is the error (standard deviation) on the response measurements of the pressure p. Also here, another criterion is possible, similar to the alternative of formula (12) [25]:

$$
\left(\sigma_{i}+\ldots+\sigma_{n}\right) \leq \frac{\left\|\sigma_{p}\right\|_{e}}{\|\hat{\mathbf{p}}\|_{e}} \sigma_{1}
$$

In [25], a method was developed that takes both errors in the FRF matrix as errors 
in the response measurements into account, which yields better results than only considering the errors in one.

\subsubsection{Other approaches}

Tikhonov manipulates singular values using a regularization parameter $\boldsymbol{b}$, [27,15]. There are several ways to determine the parameter $\boldsymbol{b}$ : ordinary cross validation $[28,29]$, general cross validation $[29,30,31]$ and the L-curve method $[32,33,34]$ being the most widely used.

Iterative inversion also can be applied as a regularization technique. Recursive Wiener filtering begins with a realistic solution [35]. A more general technique is described in [36] and [37], in which the iterative weighting of singular values is accomplished by use of a convergence parameter and an iteration number. A major drawback of iteration is the long calculation time required.

\subsubsection{Applied techniques}

In total, ten methods were considered in this study. The most effective methods are summarised in Table 1.

Table 1. Used methods to avoid matrix inversion problems and corresponding labels as used in the following figures.

\begin{tabular}{lc}
\hline Method & Label \\
\hline None & $\mathrm{N}$ \\
Over-determining with 5 positions Xi for the pressure instead of 3 & $\mathrm{O}$ \\
Elimination of the smallest singular value & $\mathrm{S} 1$ \\
Elimination of singular values smaller than 10\% of the highest singular value & $\mathrm{S} 3$ \\
Elimination of singular values of which the sum of the concerned value and all & \\
smaller singular values is smaller than a measure of the errors in the response [25] & S9
\end{tabular}

\section{EXPERIMENTAL SETUP}

\subsection{Installation and floor}

Inverse methods were applied to the case of a vibrating platform, used for muscle training in fitness centres and for physical therapy in clinics. The installation generates vertical vibrations with amplitudes up to $3 \mathrm{~mm}$. The frequency range of operation is 20 $60 \mathrm{~Hz}$, adjustable in steps of $1 \mathrm{~Hz}$. The vibrations are created by two motors with eccentric masses on their axes that contra-rotate, producing a dominant vertical vibration. The acceleration spectra show a peak at the rotation frequency and at higher harmonics, due to minor imperfections in the motor mechanism, causing friction: between rotor and stator, inside ball bearings and due to mechanical looseness in general [38].

The floor, on which the vibrating platform was placed, was of $74 \mathrm{~mm}$ reinforced concrete, with a $25 \mathrm{~mm}$ epoxy mortar and epoxy based self-levelling layer of $3 \mathrm{~mm}$. The floor was mounted in the aperture of a transmission suite. Figure 2 shows the vibrating platform (sections and plan also shown) on the concrete floor in the upper room. It must be noted that the relation between the size of the opening $\left(2 \times 2 \mathrm{~m}^{2}\right)$ and the volume of 
the transmission rooms $\left(87 \mathrm{~m}^{3}\right)$, is not the same as in real buildings, where the floors are generally (slightly) larger and the room volumes (slightly) smaller.
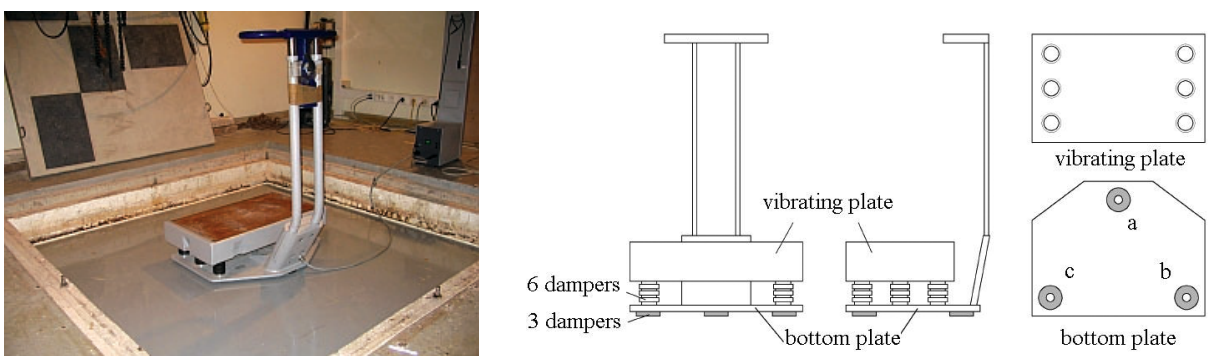

Figure 2. Vibrating platform on concrete floor and construction details with labeled contact points.

Operating frequencies of $24 \mathrm{~Hz}, 40 \mathrm{~Hz}$ and $60 \mathrm{~Hz}$ were chosen. In addition, a fourth broadband excitation was created by tapping the bottom plate with an impact hammer (see Figure 3). The force registered by the force sensor of the impact hammer was used to normalize the response. Because the resulting signals are transient and not stationary, the spectral density had to be calculated instead of a power spectrum.

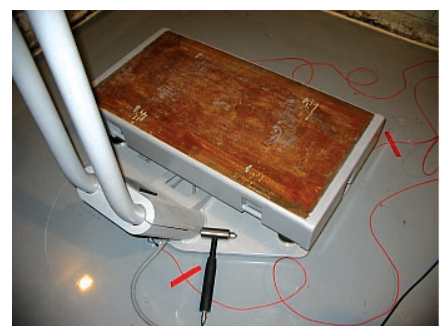

Figure 3. Excitation points on the bottom plate of the vibrating platform, using an impact hammer.

The response pressures were recorded in the lower room. The volume acceleration $Q$ was generated by a prototype low-frequency volume acceleration sound source (Figure 4). Point source behaviour is a necessary condition for the approach. However, as the source consists of two large loudspeakers, driven in phase, this condition is attained only at low frequencies and at a certain distance from the source. The source included a sensor that provided a signal proportional to the volume acceleration. The sensitivity of the sensor was determined in earlier measurements in semi-free field conditions. The source was located such that its geometrical centre, which is also judged to be the acoustical centre, coincides with the positions $X_{i}$. 


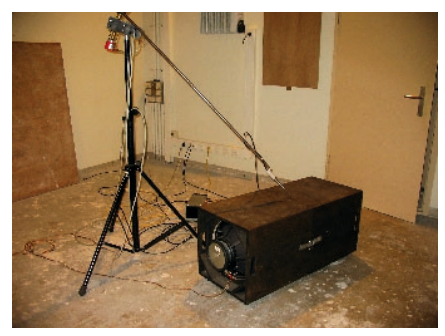

Figure 4. Volume acceleration sound source in lower transmission room.

\subsection{Direct measurement of force and velocity}

The force was also measured directly, for comparison with the inversely measured values. Force sensors were inserted between each contact point. Each force sensor was sandwiched between two aluminium cylinders with a diameter comparable with these of the contact points (Figure 5). The thickness of the cylinders was $20 \mathrm{~mm}$ so that the first resonance frequency of the sandwich was well above the frequency range of interest, which ranges from $50 \mathrm{~Hz}$ to $5000 \mathrm{~Hz}$ for general problems in building acoustics.
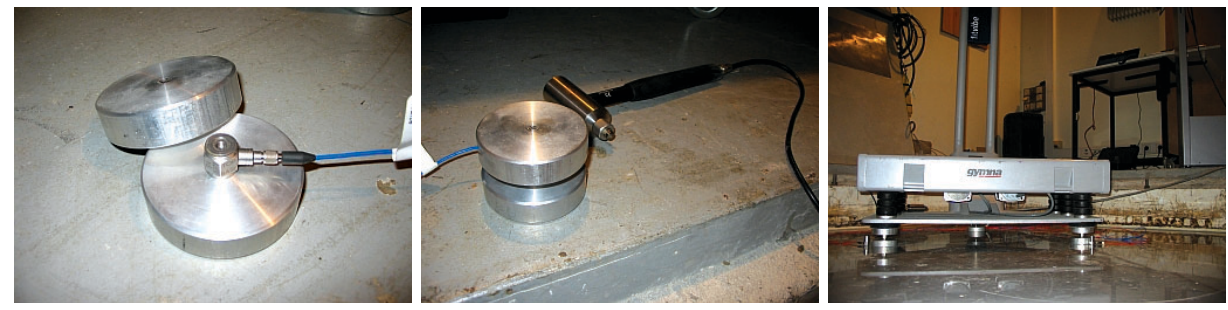

Figure 5. Mounting of the force sensors between two aluminium cylinders and arrangement under the vibrating platform.

The velocity was simultaneously recorded at positions as close as possible to the contact points.

\section{PRELIMINARY MEASUREMENTS}

\subsection{Validation of reciprocity}

Reciprocity, for the path between the concrete floor and a receiving point in the lower room, was verified. The FRF $F / p$ was determined by exciting the floor with an impact hammer, which registered the force, while the pressure in the room below measured simultaneously. The FRF $a / Q$ was measured by means of a swept-sine signal. In Figure 6 , the absolute values of both FRFs are compared. For frequencies above $180 \mathrm{~Hz}$, the assumption of a point source is violated, which explains the larger deviations. 


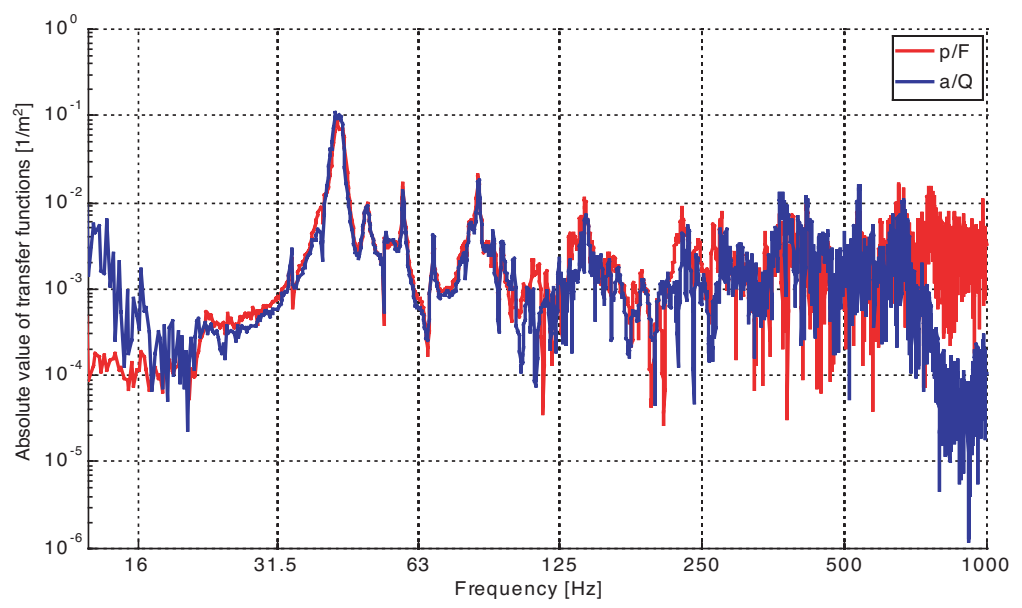

Figure 6. Absolute values of the FRFs $p / F$ and $a / Q$ for the path between a point on the concrete floor and a point in the lower room.

Direct measurement of the FRF $p / F$ could be used for inverse determination of the contact forces. The reciprocally measured FRF $a / Q$ is preferred when access to the contact points is difficult, which is often the case for machinery installations. It is also better to use reciprocity when the surface at the contact points is brittle. An impact can locally deform the surface prior to exciting the whole building element. Reciprocity is also appropriate for multiple support locations and directions, because then all supportdirections can be measured simultaneously.

\subsection{Effectiveness of over-determination}

As already mentioned, over-determination can be applied as a first step when the condition number of the FRF matrix $\mathbf{H}$ is (expected to be) high. For the three contact points, five response points were chosen in the lower room instead of the minimum of three. $\mathbf{H}$ becomes a $(5,3)$-matrix. The condition number of the $(3,3)-,(4,3)$ - and $(5,3)$-matrix is shown in Figure 7. A condition number larger than for instance 50 indicates ill-conditioning. The condition number is unfavourably large at the lowest resonance frequencies of the floor and the room (both around $45 \mathrm{~Hz}$ ). This is due to matching between points on the floor or in the room, independent of distance between them. There is a significant improvement when four points are taken. However the improvement is less obvious when five are taken. In general, over-determination is not effective at the first resonance frequency, as confirmed elsewhere $[16,17]$.

\section{COMPARISON OF DIRECTLY AND INVERSELY MEASURED FORCE}

Figure 8 compares the inversely measured force with the directly measured force at contact point $a$ when the vibrating platform is operating at a frequency of $24 \mathrm{~Hz}$. Method S3 was used to improve the condition number at the inversion. This is effective between 20 and $60 \mathrm{~Hz}$, the frequency range that corresponds with the first eigenmode of the system floor-room as can be concluded from Figure 7. Closer inspection of 
method S3 showed there was only one singular value maintained in the calculation of the inverse FRF matrix as the other singular values were smaller than $10 \%$ of the largest value. This is logical, since only one singular value is needed to describe a system with one eigenmode. It is therefore advisable to only use one singular value in the vicinity of the first eigenmode of a system. The overestimation of the inversely measured force between the harmonic peaks is due to noise in the pressure measurement.

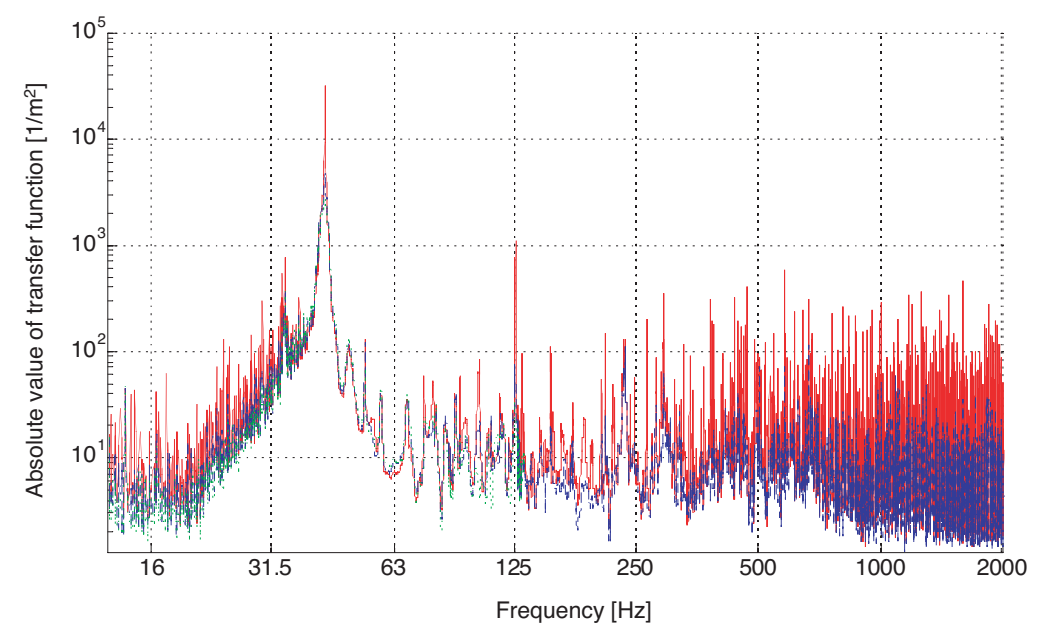

Figure 7. Condition number of the FRF matrix $H$ for 3, 4 and 5 response points in the lower room. Condition numbers greater than 50 (light blue line) indicate ill-conditioned matrices.

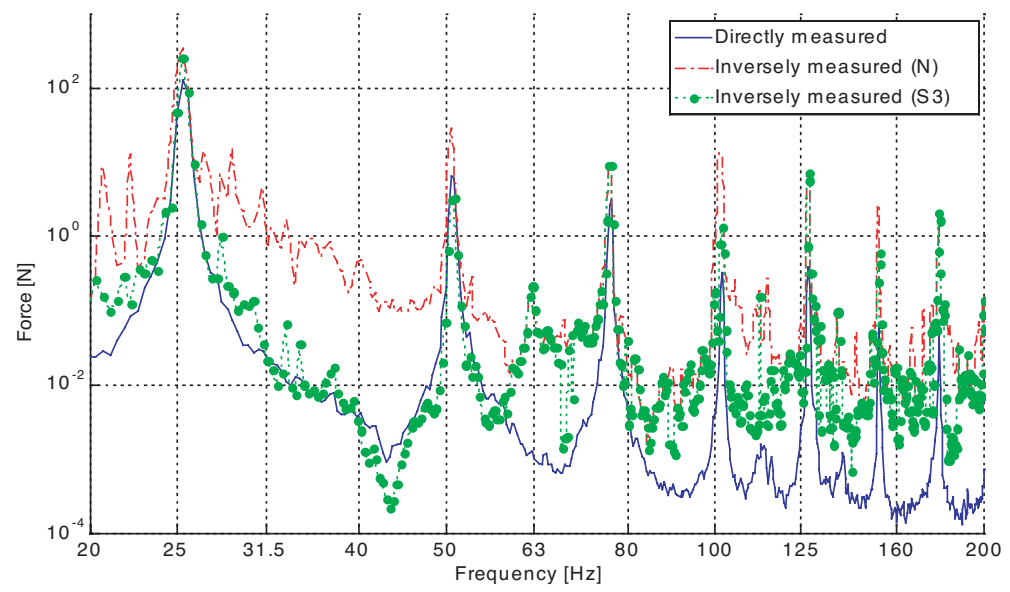

Figure 8. Directly and inversely measured force at contact point $a$ at $24 \mathrm{~Hz}$. Also shown is the result using S3. 
In Figure 9, results are shown in third-octave bands. Method S3 gives good estimates at higher frequencies, compared with the case of no application of improvement techniques. Above $800 \mathrm{~Hz}$ the volume acceleration sound source no longer behaves as an omnidirectional point source with known volume acceleration, which explains the poor agreement at the highest frequencies.

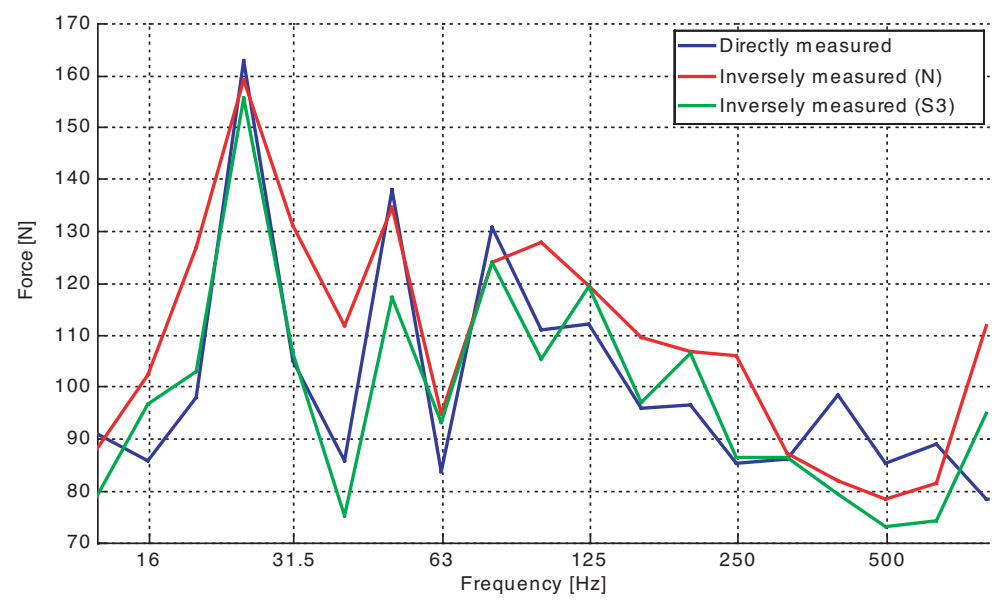

Figure 9. Directly and inversely measured force at contact point $a$ at $24 \mathrm{~Hz}$. Also shown is the inversely measured force with improvement of the inversion through method S3.

Results for the broad-band impact of the bottom plate are shown in Figure 10. Contrary to the tonal operating conditions, method $\mathrm{O}$ was used in combination with methods S1 and S9. Over-determination is effective at frequencies except in the vicinity of the first eigenmode. This supports the findings of Thite $[16,17,39]$.

Eliminating one singular value (and keeping two) is not sufficient at the first eigenmode (method S1), as explained earlier in this paper. Method S3 does not perform well in this case. Basing the singular value eliminating criterion to errors in the pressure response however delivered good results as the signal-to-noise ratio of the measured pressure is lower (method S9).

Figure 11 shows the total force, summed over the 3 contact points. Both the matrix formulation (eqn (3)) as well as the scalar formulation (eqn (6)) were used to inversely determine the measured force. As expected, the scalar formulation, that looks at the vibrating platform as if having only one contact point, works well. The matrix inversion does not work well without improvement. Over-determination gives improvement, as in the case of the force in contact point $a$ in Figure 10. A result resembling the success of the scalar formulation is attained when over-determining and singular value elimination according to method S1 (elimination of lowest singular value) are applied. This again demonstrates the power of matrix inversion improvement techniques. 


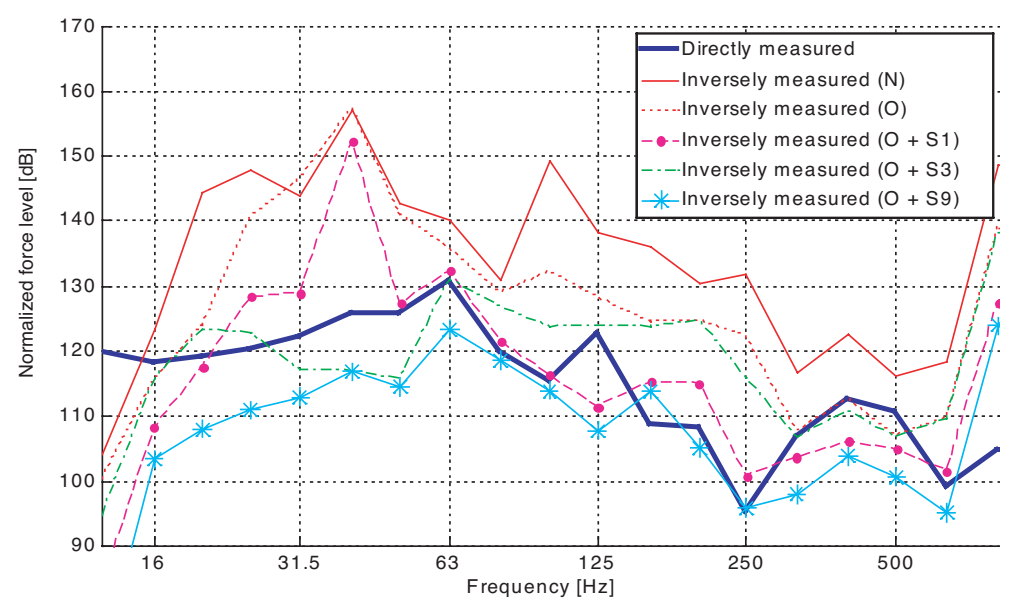

Figure 10. Directly and inversely measured force spectral density level at contact point $a$, with broadband excitation. Also shown is the inversely measured force with improvement of the inversion through method S3.

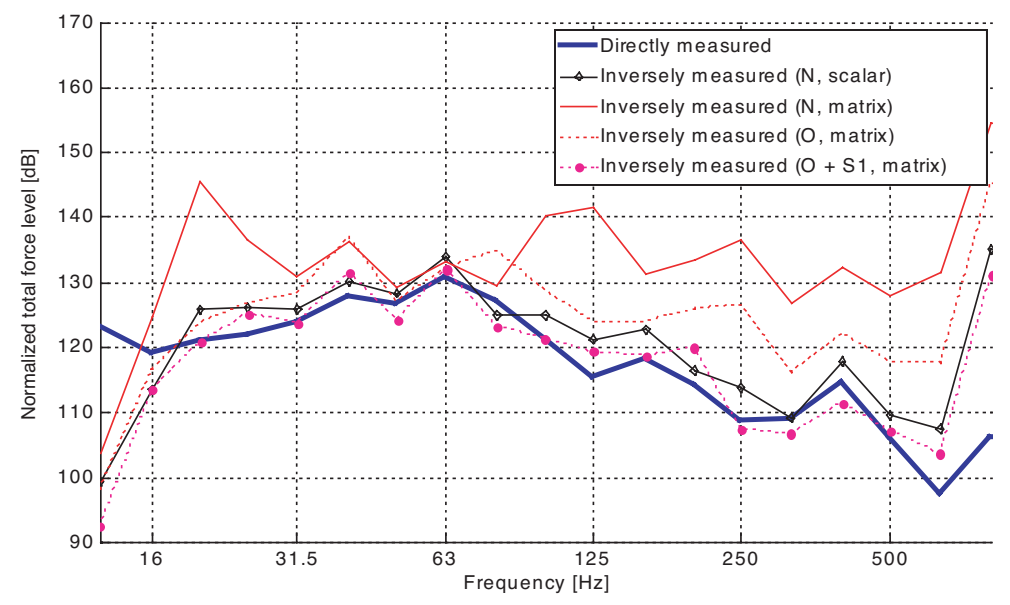

Figure 11. Directly and inversely measured total force spectral density level with broadband excitation.

\section{STRUCTURE-BORNE SOUND POWER}

Since the velocity was measured simultaneously with the pressure, the injected structure-borne sound power was obtained according to eqn (5), both with directly and inversely measured forces. The measured space-averaged sound pressure level in the lower transmission room was compared with the predicted sound pressure level according to EN 12354-5 with the injected structure-borne sound powers as input [1]. This is 


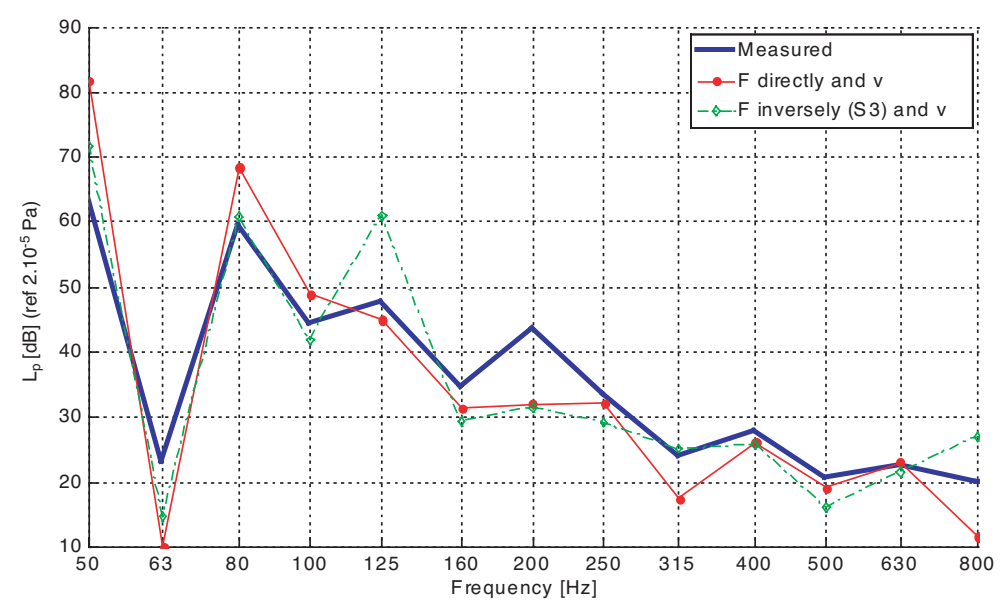

Figure 12. Measured sound pressure level and predicted value using EN 12354-5, for an operating frequency of $24 \mathrm{~Hz}$.

done because powers cannot be measured directly. When the correspondence of measured and predicted pressures is good, the injected structure-borne sound power can also be believed to be correct. In that case, the transmission path invoking the largest power into the building element can be found and possible qualitative measures can be taken to diminish the power through this transmission path. Results of compared sound pressures are shown in Figure 12 for an operating frequency of $24 \mathrm{~Hz}$.

The predicted pressures, calculated with the force and velocity as input parameter, are similar except when the force is determined inversely without improvement (not shown in the figure). This again demonstrates the usefulness of improvement techniques. However, discrepancies of $10 \mathrm{~dB}$ are observed. It must be noted that the calculation procedure in EN 12354-5 involves a lot of parameters and assumptions that cannot be detailed in this paper, but that can cause certain deviations between measured and predicted quantities. In any case, the prediction is difficult in the lower frequencies because the calculation procedures are based on statistical energy analysis, which is only valid in conditions of reasonable modal overlap [40]. This is obviously not the case in the lower frequency bands, since the lowest eigenmode of the system floor-room is situated around $40 \mathrm{~Hz}$.

In Figure 13, the same is plotted as in Figure 12, but then for the operating frequency $40 \mathrm{~Hz}$. At this excitation frequency, a strong resonance phenomenon was observable as the first eigenmode of the system lies closely to this excitation frequency (see Figure 6 and Figure 7). The similarity between the results with the directly measured and the inversely measured forces is however lost, especially for frequencies higher than $125 \mathrm{~Hz}$. This is due to dominating airborne sound transfer between the bottom plate and the floor that is relatively stronger than structure-borne sound transmission [39]. Indeed will this large, hard and flat bottom plate radiate a lot of sound to nearby surfaces when 
in strong resonance and this energy transfer is not included in the strictly structureborne model. Because of this energy transfer, the floor vibrates more than only through structure-borne sound excitation. The floor radiates the sound into the lower transmission room, where a pressure is measured that is not exclusively based on structureborne sound transmission. When back-calculating the inversely measured force out of this pressure, an overestimation of the directly measured force is the result. Finally a higher sound pressure level is calculated than this using the directly measured force because of this overestimated force. However, since the measured pressure naturally contains this airborne sound transfer component, the resemblance is better for the predicted pressures that use the inversely measured force!

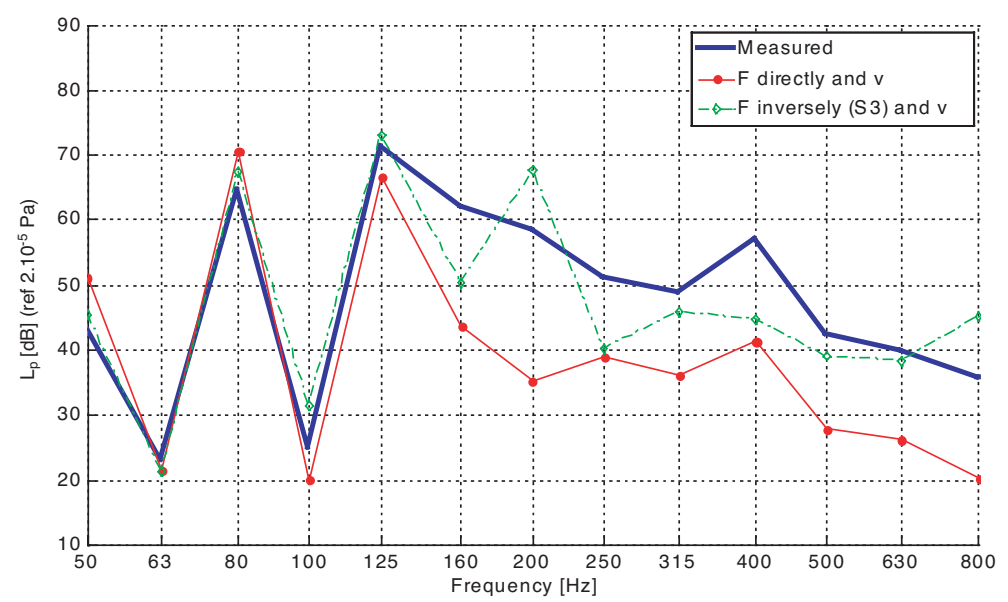

Figure 13. Measured sound pressure level and predicted using EN 12354-5, for the operating frequency of $40 \mathrm{~Hz}$.

Finally, in Figure 14 results are shown for the broadband excitation. The prediction via the directly measured force is the best approximation. There is an overestimation by the methods that use the inversely measured forces, which is thought to be due to noise in the original response pressures. The measured pressures in the lower room were indeed much smaller with the broadband operating condition than with the tonal operating conditions. Also the calculated pressure based on the scalar version of eqn (5) is used with a total inversely measured force and an averaged velocity over the contact points. Apart from a deviation in the frequency band of $50 \mathrm{~Hz}$ because of low modal density, the correspondence with the measured pressure is good up to $160 \mathrm{~Hz}$. For higher frequencies, phase differences between the contact points make a scalar formulation not feasible.

At first sight, the usefulness of predicting sound pressures using a method that makes use of this very same sound pressure seems of course very limited. However, one should not forget that the injected structure-borne sound power can also be used to determine the sound pressure in any other room of the building, provided that the acoustic properties of the building structure between the source space and the receiver space as used in EN 12354-5 are known. 


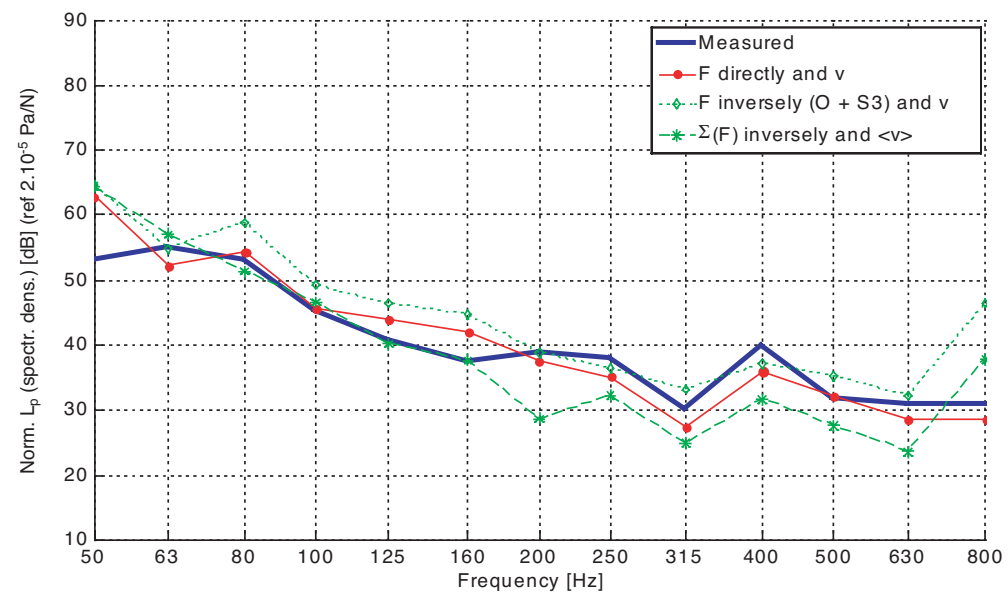

Figure 14. Measured sound pressure level and predicted using EN 12354-5, for broad-band excitation.

\section{CONCLUSIONS}

In order to predict the structure-borne sound pressure in a room of a building caused by an installation in another room of the building, as described by EN 12354-5, the injected structure-borne sound power is required. A simple way to quantify this power, is by direct multiplication of simultaneously measured velocity and force at the contact points. An inverse structural-acoustical method involving the principle of reciprocity can be used to avoid the difficult direct force measurement. The method requires a volume acceleration sound source, which is strong enough to generate measurable acceleration responses at the vibration source (or machinery) supports. The prototype source used had good low frequency characteristics appropriate for the case studied. Also a sound pressure has to be measured, but this only needs to be done in the positions where the volume acceleration sound source is placed. The sound pressure in any room of the building can then be determined if the acoustic properties of the building structure between source and receiver space are known. When the correspondence between measured and predicted pressures is good, the injected structure-borne sound power can be believed to be correct and qualitative measures can be undertaken by changing the most critical structure-borne sound transmission path.

As the FRF matrix has to be inverted in the process, several matrix inversion improvement techniques are applied to avoid problems with high condition numbers. These are based on over-determining the system and on eliminating singular values of the FRF matrix. It must be noted that this experiment on a compact piece of floor in a more or less rigid boundary is a worst case for the inversion. For one contact point, a scalar formulation can very accurately determine the total force, which is often sufficient at lower frequencies.

At comparing the inversely measured forces with the directly measured forces, several points of interest come up that have to be treated with vigilance. First, transfers paths are possible, other than those represented by the FRFs in the FRF matrix. They 
can of a structure-borne kind through a degree of freedom not included in the model, or they can be airborne. The latter can be problematic when the structure-borne sound source has a large, flat plate close to the surface of the building element. These other paths will yield an overestimation of the directly measured force. Secondly, the pressure measured to calculate the inversely measured forces must not be contaminated with noise, since this also yields an overestimation of the directly measured force. Thirdly, over-determination is a good first step but cannot improve the situation at the first eigenmode of the system. Eliminating all singular values except one is a necessary tool in the vicinity of the first eigenmode. Eliminating the smallest singular value or eliminating all singular values smaller than a certain percentage of the highest singular value are also appropriate methods. When the response is noisy, eliminating singular values based on errors in the response also can help.

Comparison of the measured pressures in the room beneath the installation with the predicted pressures using directly and inversely measured forces, shows that the disadvantage of the risk using the inverse method when also airborne sound transfer is involved, is now transformed into an advantage! This is because the predictions with directly measured forces will underestimate the measured sound pressure that includes the airborne sound transfer component. The conclusion is that the structural-acoustical inverse measurement of the force is beneficial compared to the direct measurement of the force when using this force in the calculation of a sound pressure prediction. However, one has to be aware that the inversely measured forces might not be the actual forces. Rather they are some kind of pseudo-forces in the case of other important power transfers than only transversal structure-borne ones. The result is a better prediction of the pressures when they are multiplied with the measured velocities than by using directly measured forces, if even available.

The reciprocal-inverse approach allows in-situ determination of interface forces and power transmission at very low frequencies, and without interference in the supporting structure of the vibration source or machinery, which is a considerable advantage. But, when used to quantify and compare the injected structure-borne sound power, the results should be used with great care because the inverse estimated power will not always reflect the real power transmission.

\section{ACKNOWLEDGEMENTS}

The authors would like to acknowledge the help of the company Fitvibe providing the vibrating platform. Also Qsources is gratefully thanked for providing the prototype volume acceleration sound source. Finally the financial help of the Belgian Ministry of Economic Affairs is greatly appreciated.

\section{REFERENCES}

1. EN 12354-5:2009, Building acoustics - Estimation of acoustic performance of building from the performance of elements - Part 5: Sounds levels due to the service equipment, CEN European Committee for Standardization, 2009.

2. Yap, S.H. and Gibbs, B.M., Structure-borne sound transmission from machines in buildings, part 1: Indirect measurement of force at the machine-receiver interface of a single and multi-point connected system by a reciprocal method, Journal of Sound and Vibration, 1999, 222, 85-98. 
3. Scheck, J., Fischer, H.-M. and Gibbs, B.M., Direct and indirect methods to assess the structure-borne power transmission into receiving structures, in: Proceedings of the 19th International Congress on Acoustics, 2007.

4. Helmholtz, H., Theorie des Luftschwingungen in Röhren mit offenen Enden, Journal für die reine und angewandte Mathematik, 1860, 57, 1-72.

5. Lord Rayleigh, Some general theorems relating to vibration, Proceedings of the London Mathematical Society, 1871, 4, 357-368.

6. ten Wolde, T., Reciprocity measurements in acoustical and mechano-acoustical systems, Review of theory and applications, Acta Acustica united with Acustica, 2010, 96, 1-13.

7. Ballantine, S., Reciprocity in electromagnetic, mechanical, acoustical, and interconnected systems, Proceedings of the Institute of Radio Engineers, 1929, 17, 929-951.

8. Lyamshev, L.M., A question in connection with the principle of reciprocity in acoustics, Soviet Physics Doklady, 1959, 4, 406-409.

9. Chertock, G., General reciprocity relation, Journal of the Acoustical Society of America, 1962, 34, 989.

10 1ten Wolde, T., On the validity and application of reciprocity in acoustical, mechano-acoustical and other dynamical systems, Acustica, 1973, 28, 23-32.

11. van der Linden, P.J.G., Proost, G. and Nijs, G., On efficient reciprocal measurement of vehicle body airborne isolation and vibro-acoustic transfer behavior, in: Proceedings of the 27th ISMA International Conference on Noise and Vibration Engineering, 2002, 1809-1815.12. Geebelen, N., and Vermeir, G., Reciprocity as an analysing technique in building acoustics, NAG Journaal, 2005, 175.

13. EN 15657-1:2009, Acoustic properties of building elements and of buildings Laboratory measurement of airborne and structure borne sound from building equipment - Part 1: Simplified cases where the equipment mobilities are much higher than the receiver mobilities, taking whirlpool baths as an example, CEN European Committee for Standardization, 2009.

14 Blau, M., Error considerations in inverse force synthesis: what is different with respect to sole measurements of FRFs and response spectra, in: Proceedings of Euronoise 98, 1998, 405-410.

15. Nelson, P.A., and Yoon, S.H., Estimation of acoustic source strength by inverse methods - part 1: conditioning of the inverse problem, Journal of Sound and Vibration, 2000, 233, 643-668.

16. A. N. Thite and D. J. Thompson, The quantification of structure-borne transmission paths by inverse methods, Part 1: Improved singular value rejection methods, Journal of Sound and Vibration, 2003, 264, 411-431.

17. Thite, A.N. and Thompson, D.J., Selection of response measurement locations to improve inverse force determination, Applied Acoustics, 2006, 67, 797-818.

18. Blau, M., Force spectra identification by FRF matrix inversion: a sensor placement criterion, Journal of the Acoustical Society of America, 1999, 105, 970 (A). 
19. Veronesi, W.A. and Maynard, J.D., Digital holographic reconstruction of sources with arbitrarily shaped surfaces, Journal of the Acoustical Society of America, 1989, 85, 588-598.

20. Golub, G.H. and Van Loan, C.F., Matrix computations, The John Hopkins University Press, Baltimore, 1988.

21. Moorhouse, A.T., Compensation for discarded singular values in vibro-acoustic inverse methods, Journal of Sound and Vibration, 2003, 267, 245-252.

22. Romano, J. and López, J.A., Practical application of transfer path analysis to resolve structure-borne noise problems in vehicle design, in: Proceedings of the 21 st ISMA International Conference on Noise and Vibration Engineering, 1996.

23. Powell, R.E., Multichannel inverse filtering of machinery vibration signals, M.I.T. Massachusetts Institute of Technology, 1983.

24. Janssens, M.H.A. and Verheij, J.W., The use of an equivalent forces method for the experimental quantification of structural sound transmission in ships, Journal of Sound and Vibration, 1999, 226, 305-328.

25. Thite, A.N. and Thompson, D.J., A systematic evaluation of different methods to improve inverse force determination, in: Proceedings of Euronoise 2003, 2003.

26. Janssens, M.H.A., Verheij, J.W. and Loyau, T., Experimental example of the pseudo-forces method used in characterisation of a structure-borne sound source, Applied Acoustics, 2002, 63, 9-34.

27. Tikhonov, A.N., Solution of incorrectly formulated problems and the regularisation method, Soviet Physics Doklady, 1963, 4, 1035-1038.

28. Allen, D.M., The relationship between variable selection and data augmentation and a method for prediction, Technometrics, 1974, 16, 125-127.

29. Yoon, S.H. and Nelson, P.A., Estimation of acoustic source strength by inverse methods - part 2: experimental investigation of methods for choosing regularization parameters, Journal of Sound and Vibration, 2000, 233, 669-705.

30. Yoon, S.H., Reconstruction of acoustic source strength distributions and their interactions by inverse techniques, $\mathrm{PhD}$ Thesis, University of Southampton, 1998.

31. Kim, Y., Spatial resolution limits for the reconstruction of acoustic source distribution by inverse techniques, University of Southampton, 2002.

32. Kim, Y. and Nelson, P.A., Optimal regularisation for acoustic source reconstruction by inverse methods, Journal of Sound and Vibration, 2004, 275, 463-487.

33. Hansen, P.C. and O'Leary, D.P., The use of the L-curve in the regularisation of discrete ill-posed problem, SIAM Journal on Scientific Computing, 1993, 14, 1487-1503.

34. Hansen, P.C., Rank-deficient and discrete ill-posed problems: numerical aspects of linear inversion, Society for Industrial and Applied Mathematics, Philadelphia, 1998.

35. Bai, M.R., Acoustical source characterization by using recursive Wiener filtering, Journal of the Acoustical Society of America, 1995, 97, 2657-2663. 
36. Thite, A.N., Inverse determination of structure-borne sound sources, $\mathrm{PhD}$ Thesis, University of Southampton, 2002.

37. Thite, A.N., and Thompson, D.J., The quantification of structure-borne transmission paths by inverse methods, Part 2: Use of regularization techniques, Journal of Sound and Vibration, 2003, 264, 433-451.

38. Bate, G.H., Vibration diagnostics for industrial electric motor drives, Brüel and Kjaer, Naerum, Denmark, 1980.

39. Schevenels, P., Dijckmans, A., van der Linden, P.J.G. and Vermeir, G., Identification of structure-borne sound paths of service equipment in buildings using structural-acoustic reciprocity, in: Proceedings of the 16th International Congress of Sound and Vibration, 2009.

40. Hopkins, C., Sound insulation, Butterworth-Heinemann, Elsevier Ltd., Oxford, United Kingdom, 2007. 
